\title{
Effect of Antioxidant Mineral Elements Supplementation in the Treatment of Hypertension in Albino Rats
}

\author{
S. A. Muhammad, ${ }^{1}$ L. S. Bilbis, ${ }^{1}$ Y. Saidu, ${ }^{1}$ and Y. Adamu ${ }^{2}$ \\ ${ }^{1}$ Biochemistry Department, Usmanu Danfodiyo University, PMB, Sokoto 2346, Nigeria \\ ${ }^{2}$ Faculty of Veterinary Medicine, Usmanu Danfodiyo University, PMB , Sokoto 2346, Nigeria
}

Correspondence should be addressed to S. A. Muhammad, salhajimuhammad@yahoo.com

Received 15 April 2012; Accepted 21 May 2012

Academic Editor: Adrian Manea

Copyright ( 92012 S. A. Muhammad et al. This is an open access article distributed under the Creative Commons Attribution License, which permits unrestricted use, distribution, and reproduction in any medium, provided the original work is properly cited.

\begin{abstract}
Oxidative stress has been implicated in various pathologies, including hypertension, atherosclerosis, diabetes, and chronic renal disease. The current work was designed with the aim of investigating the potentials of antioxidants copper, manganese, and zinc in the treatment of hypertension in Wistar rats. The rats were fed $8 \% \mathrm{NaCl}$ diet for 5 weeks and treatment with supplements in the presence of the challenging agent for additional 4 weeks. The supplementation significantly decreased the blood pressure as compared with hypertensive control. The result also indicated significant decreased in the levels of total cholesterol, triglyceride, low-density lipoprotein cholesterol and very low-density lipoprotein cholesterol, malondialdehyde, insulin and increase in the high-density lipoprotein cholesterol, total antioxidant activities, and nitric oxide of the supplemented groups relative to the hypertensive control. The average percentage protection against atherogenesis indicated $47.13 \pm 9.60 \%$ for all the supplemented groups. The mean arterial blood pressure showed significant positive correlation with glucose, total cholesterol, triglyceride, low-density lipoprotein cholesterol, very low-density lipoprotein cholesterol, atherogenic index, insulin resistance and malondialdehyde while high density lipoprotein-cholesterol and total antioxidant activities showed negative correlation. The result therefore indicated strong relationship between oxidative stress and hypertension and underscores the role of antioxidant minerals in reducing oxidative stress, dyslipidemia, and insulin resistance associated with hypertension.
\end{abstract}

\section{Introduction}

Hypertension is one of the most common diseases worlds over and a major cause of death from cardiovascular failure. Due to associated morbidity and mortality, hypertension is a public health problem [1], and thus the need to search for proper preventive and management strategies should be the concern of health care providers. Increased vascular oxidative stress could be involved in the pathogenesis of hypertension $[2,3]$ a major risk factor for cardiovascular disease and mortality in the developed and developing countries. The onset of hypertension is caused by complex interactions between genetic predisposition and environmental factors [4]. Increased salt intake may aggravate the rise in blood pressure and the development of consequential end-organ damage [5].
The novel concept that structural and functional abnormalities in the vasculature, including endothelial dysfunction, increased oxidative stress, and decreased antioxidant activities, may antedate hypertension and contribute to its pathogenesis, has gained support in recent years [6]. Animal studies have generally supported the hypothesis that increased blood pressure is associated with increased oxidative stress [7]. Elevated lipid peroxidation byproducts and decreased activity of antioxidant systems have been reported in hypertensive subjects [8]. Several studies have indicated an increase in $\mathrm{O}_{2}{ }^{-}$levels in hypertension [9] and implicate NADPH oxidase as a source of excess $\mathrm{O}_{2}{ }^{-}[10,11]$.

Angiotensin II has been shown to be a potent activator of NADPH oxidase activity in vascular smooth muscle, endothelial cells, and cardiomyocytes [12]. A common finding in all types of hypertension as well as diabetes and 
metabolic syndrome is endothelial dysfunction, characterized by an imbalance in the expression of and sensitivity to vasodilator and vasoconstrictor agents resulting in increased vascular tone and thus an increase in resistance to flow $[13,14]$.

Copper, manganese, and zinc are commonly referred to as antioxidant minerals that are required for the activity of some antioxidant enzymes. Hiroyuki et al. [15] reported that zinc deficiency might play a crucial role in the development of genetic hypertension presumably through the oxidative stress caused by hypertension. It is known that superoxide anion rapidly inactivates the endothelium-derived vasodilator, nitric oxide, thereby promoting vasoconstriction [16]. Consequently, attempts to counteract the hypertensive effects of reactive oxygen species have led to the use of exogenous antioxidants to improve vascular function and reduce blood pressure in animal models [17] and human hypertension $[18,19]$. Thus, evaluation of potential effect of antioxidants copper, manganese, and zinc in the management of hypertension form the basis of this study.

\section{Methods}

2.1. Chemicals and Reagents. Analytical graded chemicals and reagents were used for this research. Copper sulphate and manganese sulphate were sourced from May and Baker, England, while zinc sulphate was from J.T. Baker chemical company, Philipsburg, New Jersey.

2.2. Experimental Animals. Male wistar rats weighing between 150-180 g were purchased from the Faculty of Veterinary Medicine, Usmanu Danfodiyo University, Sokoto, Nigeria and were allowed to acclimatize for two weeks before the commencement of the experiment. The animals were grouped into six groups of 5 rats each and they were fed pelletized growers' feed (Vital feed, Jos, Nigeria) and allowed access to water ad libitum throughout the experimental period. The experimental protocol was approved by the Ethical Committee of the Usmanu Danfodiyo University, Sokoto, Nigeria.

2.3. Induction of Hypertension. The rats were placed on a high-salt diet $(8 \% \mathrm{NaCl})$ except normotensive control for 9 weeks by adding $8 \% \mathrm{NaCl}$ to the feed [20]. Treatment commenced from the 6 th week of salt loading.

2.4. Measurement of Blood Pressure. Blood pressure was monitored weekly by the tail-cuff method using noninvasive Ugo Basile, series 58500 Blood Pressure Recorder. Average of four readings was taken for each rat, and the temperature of the rat was monitored throughout the measurement period. Mean arterial blood pressure was calculated according to the following equation: $\mathrm{DP}+(1 / 3)(\mathrm{SP}-\mathrm{DP})$ where SP and $\mathrm{DP}$ are systolic and diastolic pressure, respectively.

2.5. Preparation of Supplements. Copper, manganese, and zinc were prepared by dissolving copper sulphate, manganese sulphate, and zinc sulphate in distilled water to obtain $2.5 \mathrm{mg} / \mathrm{mL}$ of copper, $2.4 \mathrm{mg} / \mathrm{mL}$ of manganese, and $11 \mathrm{mg} / \mathrm{mL}$ of zinc, respectively. All the supplements were prepared just prior to administration.

\subsection{Grouping of Animals and Treatment}

Group I Normal untreated /distilled water.

Group II Hypertensive control/distilled water.

Group III Salt-loaded treated with $4 \mathrm{mg} / \mathrm{kg}$ of copper.

Group IV Salt-loaded treated with $10 \mathrm{mg} / \mathrm{kg}$ of manganese.

Group V Salt-loaded treated with $20 \mathrm{mg} / \mathrm{kg}$ of zinc.

Group VI Salt-loaded treated with $4 \mathrm{mg} / \mathrm{kg}$ of copper, $10 \mathrm{mg} / \mathrm{kg}$ of manganese, and $20 \mathrm{mg} / \mathrm{kg}$ of zinc.

The concentrations of the supplements were selected based on the recommended dietary allowance and the appropriate dosages administered orally to the treated groups according to their body weight by intubation using intravenous cannula tube for 4 weeks. Twenty four hours after the last treatment, the animals were anaesthetized with chloroform vapour and fasting blood samples were collected through cardiac puncture into labelled tubes for biochemical analyses. Weight changes of the rats were monitored throughout the experimental period.

2.7. Estimation of Biochemical Parameters. The blood sample was allowed to clot and centrifuged at $4000 \mathrm{~g}$ for ten minutes and the serum obtained was used for the estimation of glucose, lipid profile, total antioxidant status, insulin, superoxide dismutase, catalase, and nitric oxide. The animals were sacrificed and the liver of each rat was dissected out, rinsed with ice-cold saline to remove the blood. 10\% homogenate was prepared in ice-cold $0.1 \mathrm{M}$ Tris buffer, $\mathrm{pH}$ 7.4 using homogenizer. The homogenate was centrifuged at $4000 \mathrm{~g}$ for 15 minutes. The supernatants were used for estimation of thiobarbituric acid reactive substance (TBARS) and glutathione peroxidase activity.

The fasting serum glucose level was estimated by glucose oxidase method [21]. Serum total cholesterol [22], triglyceride [23] and high-density lipoprotein cholesterol [24] were determined by enzymatic method.

Serum low-density lipoprotein cholesterol and very low density lipoprotein cholesterol was calculated by the formula of friedewald et al. [25]. Atherogenic index was calculated as the ratio of LDL cholesterol to HDL cholesterol [26].

Colorimetric method was used for the estimation of serum total antioxidant status [27] and tissue malondialdehyde [28].

Cayman's Superoxide Dismutase Assay Kit was used for the estimation of SOD. The assay utilizes a tetrazolium salt for the detection of superoxide radicals generated by xanthine oxidase and hypoxanthine at $450 \mathrm{~nm}$. One unit of SOD is defined as the amount of enzyme needed to exhibit $50 \%$ dismutation of the superoxide radicals. 
The catalase activity was estimated using Cayman's Catalase Assay Kit. The method is based on the reaction of the enzyme with methanol in the presence of an optimal concentration of $\mathrm{H}_{2} \mathrm{O}_{2}$. The formaldehyde produced is measured with 4-amino-3-hydrazino-5-mercapto-1,2,4-triazole (Purpald) as the chromogen at $540 \mathrm{~nm}$.

Glutathione peroxidase activity was assayed using Cayman's Assay Kit. This assay measures glutathione peroxidase activity indirectly by a coupled reaction with glutathione reductase. Oxidized glutathione, produced upon reduction of hydroperoxide by glutathione peroxidase, is recycled to its reduced state by glutathione reductase and NADPH. The oxidation of NADPH to $\mathrm{NADP}^{+}$is accompanied by a decrease in absorbance at $340 \mathrm{~nm}$.

Nitric oxide was estimated by Cayman's Assay Kit. The assay provides an accurate and convenient method for measurement of total nitrate/nitrite concentration in a simple two-step process. The first step is the conversion of nitrate to nitrite utilizing nitrate reductase. The second step is the addition of the Griess reagent which converts nitrite into a deep purple azo compound.
Insulin was estimated by SPI biorat insulin enzyme immunoassay kit. The assay is based on the competition between unlabelled rat insulin and acetylcholinesterase linked to rat insulin (tracer) for limited specific guineapig anti-rat insulin antiserum sites. The plate was then washed and Ellman's reagent added to the wells, and the acetylcholinesterase tracer acts on the Ellman's reagent to form a yellow compound which was determined at $405 \mathrm{~nm}$.

Insulin resistance index was calculated by Homeostasis Model Assessment-Insulin Resistance (HOMA-IR) [29].

\section{HOMA-IR \\ $=\frac{\text { Fasting glucose }(\mathrm{mmol} / \mathrm{L}) \times \text { Fasting insulin }(\mu \mathrm{U} / \mathrm{mL})}{22.5}$}

Percentage protection against atherogenesis was calculated using the following equation

Atherogenic index (AI) of Hypertensive control (HC) - AI of treated group Atherogenic index (AI) of Hypertensive control (HC)

2.8. Statistical Analysis. Values are expressed as mean \pm standard deviation for 5 rats in each group. The result were analysed statistically using one way analysis of variance (ANOVA), followed by Dunnett's multiple comparison test using GraphPad Instat software. Differences were considered significant when $P<0.05$.

\section{Results}

The weight gain of rats (Figure 1) indicated that salt-loaded untreated control gain more weight $(53.89 \pm 2.50)$ than the treated groups and normotensive control. Supplementation showed significant $(P<0.01)$ effect on the weight changes of the rats as compared to the hypertensive control.

There was significant $(P<0.05)$ increase in mean arterial blood pressure of the salt-loaded rats (Figure 2). Supplementation caused significant decrease in the mean arterial blood pressure of the treated groups relative to the hypertensive control.

The effect of mineral elements in SBP, DBP, and MABP is presented in Figure 3. The result indicated significant $(P<0.05)$ increase in the \% reduction of SBP between the minerals combined $(11.02 \%)$ group and groups supplemented with copper (7.36\%), manganese $(6.59 \%)$, and zinc $(7.73 \%)$, respectively while there was no significant $(P>0.05)$ difference in the \% reduction of DBP and MABP between the minerals combined group and copper, manganese-, and zinc- supplemented groups. The \% reduction in DBP of copper-, zinc-, and manganese-treated groups indicated $21.71 \%, 20.68 \%$, and $18.29 \%$, respectively, while mineral combined was $24.47 \%$. The group treated with
TABle 1: Effect of antioxidant minerals on glucose, insulin and insulin resistance in salt-induced hypertensive.

\begin{tabular}{lccc}
\hline Group & $\begin{array}{c}\text { Glucose } \\
(\mathrm{mmol} / \mathrm{L})\end{array}$ & $\begin{array}{c}\text { Insulin } \\
(\mu \mathrm{U} / \mathrm{mL})\end{array}$ & HOMA-IR \\
\hline I & $4.28 \pm 0.31$ & $2.98 \pm 1.42$ & $0.56 \pm 0.23$ \\
II & $6.50 \pm 0.83^{\rho}$ & $17.01 \pm 4.04^{\rho}$ & $4.81 \pm 0.68^{\rho}$ \\
III & $5.62 \pm 0.72^{\gamma}$ & $4.94 \pm 1.51^{\alpha}$ & $1.21 \pm 0.26^{\alpha}$ \\
IV & $5.66 \pm 0.75^{\rho}$ & $9.68 \pm 2.76^{\alpha, \rho}$ & $2.48 \pm 0.46^{\alpha, \rho}$ \\
V & $5.66 \pm 0.66^{\rho}$ & $11.06 \pm 4.50^{\beta, \rho}$ & $2.93 \pm 0.42^{\alpha, \rho}$ \\
VI & $4.63 \pm 0.21^{\alpha}$ & $6.56 \pm 1.47^{\alpha}$ & $1.34 \pm 0.24^{\alpha, \gamma}$ \\
\hline
\end{tabular}

HOMA-IR: Homeostasis Model Assessment-Insulin Resistance, Inormotensive control, II-hypertensive control, III-group treated with copper, IV-group treated with manganese, V-group treated with zinc, VI-group treated with all the minerals.

Values are expressed as Mean $\pm \mathrm{SD} ; n=5 .{ }^{\alpha} P<0.01$ when compared with grp II, $\beta P<0.05$ when compared with grp II, $\rho_{P}<0.01$ when compared with grp I, $\gamma P<0.05$ when compare with grp I by Dunnette's multiple comparison test.

mineral combined showed $18.52 \%$ reduction in MABP, zinc (15.38\%), and copper (14.68\%) while manganese-treated group, $13.80 \%$, was the lowest in the group.

Salt loading caused significant increase in serum glucose, insulin, and insulin resistance (Table 1), and supplementations with the mineral elements reverse the trend.

Effect of supplementation on serum lipid profile and atherogenic index is presented in Table 2. The result indicated significant decrease in the levels of TC, TG, LDL-C, VLDL-C, and AI and increase in HDL-C as compared with salt-loaded untreated group. 
TABLE 2: Effect of antioxidant minerals on lipid profile and atherogenic index.

\begin{tabular}{lcccccc}
\hline Group & TC $(\mathrm{mg} / \mathrm{dL})$ & TG $(\mathrm{mg} / \mathrm{dL})$ & HDL-C $(\mathrm{mg} / \mathrm{dL})$ & LDL-C $(\mathrm{mg} / \mathrm{dL})$ & VLDL-C $(\mathrm{mg} / \mathrm{dL})$ & AI \\
\hline I & $78.08 \pm 4.03$ & $63.89 \pm 5.17$ & $40.44 \pm 5.30$ & $24.53 \pm 4.85$ & $12.77 \pm 1.03$ & $0.61 \pm 0.16$ \\
II & $123.64 \pm 7.47^{\rho}$ & $121.10 \pm 12.14^{\rho}$ & $30.36 \pm 2.23^{\rho}$ & $69.05 \pm 5.83^{\rho}$ & $24.21 \pm 2.42^{\rho}$ & $2.27 \pm 0.20^{\rho}$ \\
III & $109.82 \pm 3.43^{\beta, \rho}$ & $70.87 \pm 4.63^{\alpha}$ & $41.45 \pm 2.74^{\alpha, \rho}$ & $54.18 \pm 3.14^{\alpha}$ & $14.17 \pm 0.92^{\alpha}$ & $1.30 \pm 0.11^{\alpha, \rho}$ \\
IV & $108.38 \pm 5.18^{\alpha, \rho}$ & $65.97 \pm 4.76^{\alpha}$ & $39.98 \pm 6.42^{\alpha}$ & $55.26 \pm 5.96^{\beta, \rho}$ & $13.18 \pm 0.95^{\alpha}$ & $1.39 \pm 0.36^{\alpha, \rho}$ \\
V & $102.19 \pm 11.64^{\alpha, \rho}$ & $83.50 \pm 8.33^{\alpha, \rho}$ & $38.05 \pm 3.12^{\beta}$ & $46.69 \pm 10.9^{\alpha, \rho}$ & $16.69 \pm 1.66^{\alpha, \rho}$ & $1.23 \pm 0.33^{\alpha, \rho}$ \\
VI & $94.02 \pm 5.24^{\alpha, \rho}$ & $80.02 \pm 12.06^{\alpha, \gamma}$ & $41.14 \pm 1.23^{\alpha}$ & $36.86 \pm 5.40^{\alpha, \gamma}$ & $16.00 \pm 2.41^{\alpha, \gamma}$ & $0.89 \pm 0.14^{\alpha}$ \\
\hline
\end{tabular}

TC: total cholesterol, TG: triglyceride, HDL-C: high-density lipoprotein cholesterol, LDL-C: low-density lipoprotein cholesterol, VLDL-C: very low density lipoprotein cholesterol, AI: atherogenic index, I: normotensive control, II: hypertensive control, III: group treated with copper, IV: group treated with manganese, V: group treated with zinc, and VI: group treated with all the minerals.

Values are expressed as Mean $\pm \mathrm{SD} ; n=5 .{ }^{\alpha} P<0.01$ when compared with group II, ${ }^{\beta} P<0.05$ when compared with grp II, ${ }^{\rho} P<0.01$ when compared with group I, and $\gamma P<0.05$ when compare with group I by Dunnette's multiple comparison test.

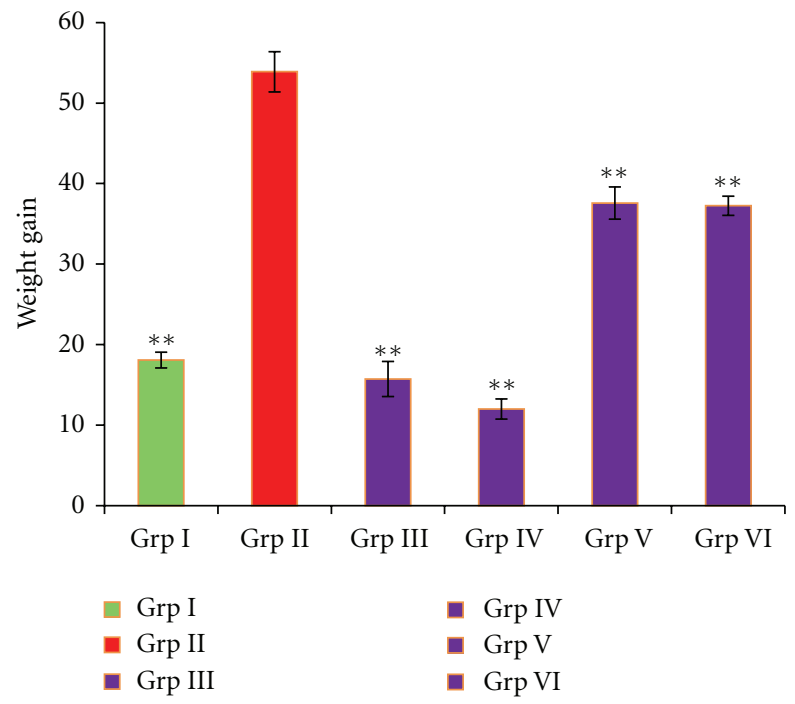

FIGURE 1: Weight gain of salt-induced hypertensive treated with antioxidant minerals. Grp I: normal untreated, Grp II: hypertensive untreated, Grp III: salt loaded treated with copper, Grp IV: salt loaded treated with manganese, Grp V: salt loaded treated with zinc, and Grp VI: salt loaded treated with minerals combined ${ }^{* *} P<0.01$ when compared with group II.

The $\%$ protection against atherogenesis (Figure 4$)$ indicated significant $(P<0.01)$ increase in the \% protection in the group supplemented with minerals combined as compared to the copper, manganese, and zinc groups. The group treated with minerals combined showed the highest protection of $60.79 \%$ while the group supplemented with manganese showed the lowest protection of $38.76 \%$.

Effect of supplementation on total antioxidant status, nitric oxide, and MDA (Table 3 ) showed significant increase in the levels of TAS between untreated group and manganese $(P<0.05)$ and mineral combined $(P<0.01)$ groups. The result also indicated significant $(P<0.01)$ decrease in the tissue MDA of the supplemented groups as compared with untreated control. Endothelial function was also improved following supplementation.

The result of effect of supplementation on antioxidant enzymes is presented in Table 4. The result indicated that

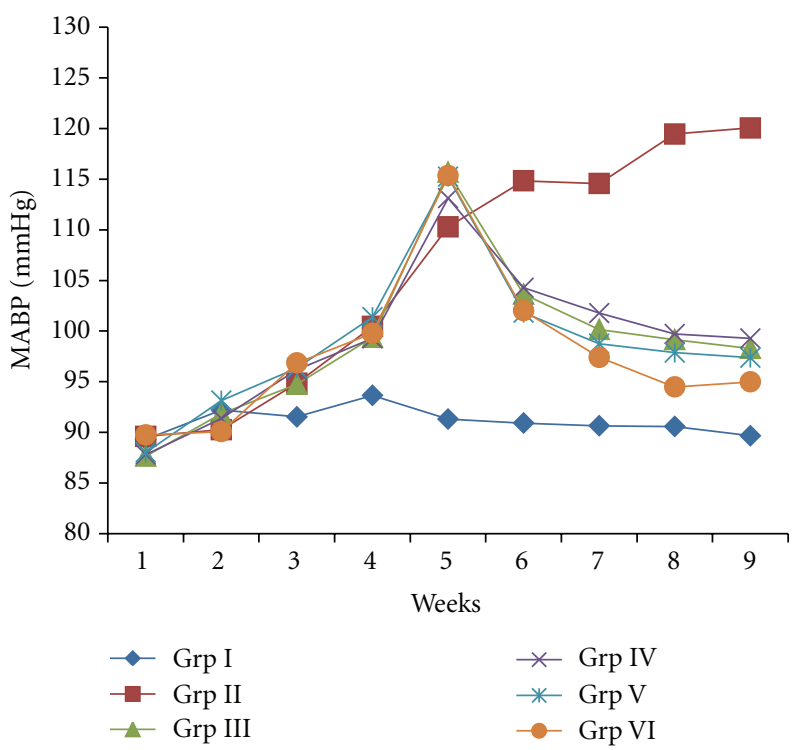

Figure 2: Effect of salt diet and antioxidant supplementation on mean arterial blood pressure of salt-loaded rats. Week 1-5: salt diet only, week 6-9: salt diet plus supplements, MABP-mean arterial blood pressure Grp I: normal untreated, Grp II: hypertensive untreated, Grp III: salt loaded treated with copper, Grp IV: salt loaded treated with manganese, Grp V: salt loaded treated with zinc, and Grp VI: salt-loaded treated with minerals combined.

supplementation increased the activities of cat, Gpx, and SOD as compared with hypertensive control.

Correlation coefficient $(r)$ of MABP against glucose, insulin resistance, lipid profile, and oxidative stress markers is presented in Figure 5. The result showed significant positive correlation between MABP and glucose, insulin, insulin resistance, TC, TG, LDL-C, VLDL-C, AI, and MDA while HDL-C, TAS, SOD, Cat, and GPx showed negative correlation with MABP.

\section{Discussion}

Hypertension is among the top most risk factors for cardiovascular disease [30]. In this model, a diet containing $8 \% \mathrm{NaCl}$ was used to induce hypertension in wistar rats 


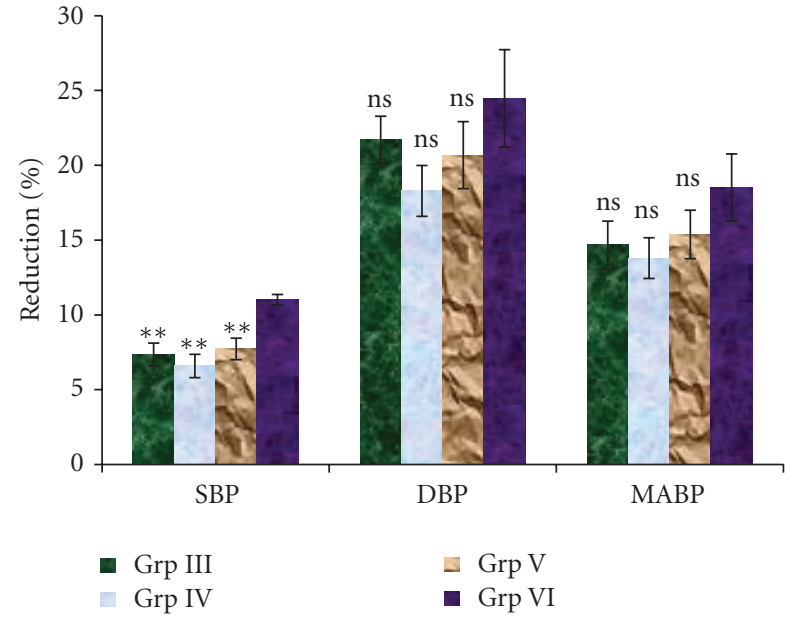

FIGURE 3: The mean percentage reduction in blood pressure of supplemented groups. SBP: systolic blood pressure, DBP: diastolic blood pressure, MABP: mean arterial blood pressure, Grp III: salt-loaded treated with copper, Grp IV: salt-loaded treated with manganese, Grp V: salt loaded treated with zinc, and Grp VI: salt loaded treated with minerals combined. ${ }^{* *} P<0.01$ and ns: not significant when compared with Grp VI.

TABle 3: Effect of antioxidant minerals on total antioxidant, nitric oxide, and lipid peroxidation.

\begin{tabular}{lccc}
\hline Group & $\begin{array}{c}\text { Nitric oxide } \\
(\mu \mathrm{M})\end{array}$ & $\begin{array}{c}\text { TAS } \\
(\mathrm{mmol} / \mathrm{L})\end{array}$ & $\begin{array}{c}\text { MDA } \\
(\mathrm{nmol} / \mathrm{mg} \text { tissue })\end{array}$ \\
\hline I & $28.14 \pm 3.85$ & $1.64 \pm 0.24$ & $0.382 \pm 0.04$ \\
II & $17.70 \pm 3.07^{\rho}$ & $0.70 \pm 0.17^{\rho}$ & $1.210 \pm 0.22^{\rho}$ \\
III & $31.55 \pm 6.16^{\alpha}$ & $0.99 \pm 0.28^{\rho}$ & $0.549 \pm 0.11^{\alpha}$ \\
IV & $22.00 \pm 5.62$ & $1.13 \pm 0.26^{\beta, \rho}$ & $0.569 \pm 0.08^{\alpha}$ \\
V- & $18.81 \pm 3.01^{\gamma}$ & $1.00 \pm 0.15^{\rho}$ & $0.545 \pm 0.11^{\alpha}$ \\
VI & $30.81 \pm 5.67^{\alpha}$ & $1.40 \pm 0.06^{\alpha}$ & $0.512 \pm 0.14^{\alpha}$ \\
\hline
\end{tabular}

TAS: total antioxidant status, MDA: malondialdehyde, I: normotensive control, II: hypertensive control, III: group treated with copper, IV: group treated with manganese, V: group treated with zinc, and VI: group treated with all the minerals.

Values are expressed as Mean $\pm \mathrm{SD} ; n=5 .{ }^{\alpha} P<0.01$ when compared with group II, ${ }^{\beta} P<0.05$ when compared with group II, ${ }^{\rho} P<0.01$ when compared with group I, and $\gamma_{P}<0.05$ when compare with group I by Dunnette's multiple comparison test.

for 5 weeks and salt-loaded diet with supplementation for additional 4 weeks. High salt has been reported to cause hypertension in rats $[20,31]$. The mechanism by which highsalt diets induced hypertension could be due to increase in the level of circulating sodium which cause cells to release water due to osmotic pressure which elevates the pressure on blood vessel walls [32]. Other possible mechanisms could be in part due to an increase in the plasma's capacity to inhibit $\mathrm{Na}^{+}, \mathrm{K}^{+}$-Adenosine Triphosphotase which raises the blood pressure by inhibiting the sodium-calcium exchange pump in vascular smooth muscle [33], or that sodium diet is associated with increased intrarenal angiotensin II [34] which may result in renal vasoconstriction and increased renal $\mathrm{O}_{2}{ }^{-}$production due to activation of NADPH oxidase.
TABLE 4: Effect of supplementation on antioxidant enzymes.

\begin{tabular}{lccc}
\hline Group & $\begin{array}{c}\text { Catalase } \\
(\mathrm{nmol} / \mathrm{min} / \mathrm{mL})\end{array}$ & $\begin{array}{c}\mathrm{GPx} \\
(\mathrm{nmol} / \mathrm{min} / \mathrm{mL})\end{array}$ & $\begin{array}{c}\text { SOD } \\
(\mathrm{U} / \mathrm{mL})\end{array}$ \\
\hline I & $26.27 \pm 4.47$ & $95.76 \pm 9.75$ & $5.21 \pm 1.46$ \\
II & $14.52 \pm 3.16^{\rho}$ & $28.01 \pm 7.26^{\rho}$ & $2.86 \pm 0.62^{\gamma}$ \\
III & $19.41 \pm 3.14^{\gamma}$ & $49.15 \pm 9.72^{\alpha, \rho}$ & $4.83 \pm 1.06^{\beta}$ \\
IV & $21.67 \pm 4.27^{\beta}$ & $49.40 \pm 7.71^{\alpha, \rho}$ & $5.13 \pm 0.94^{\beta}$ \\
V & $21.05 \pm 2.95^{\beta}$ & $47.62 \pm 9.38^{\alpha, \rho}$ & $5.82 \pm 1.31^{\alpha}$ \\
VI & $23.71 \pm 3.30^{\alpha}$ & $68.00 \pm 7.93^{\alpha, \rho}$ & $4.89 \pm 1.29^{\beta}$ \\
\hline
\end{tabular}

GPx: glutathione peroxidase, SOD: superoxide dismutase, I: normotensive control, II: hypertensive control, III: group treated with copper, IV: group treated with manganese, V: group treated with zinc, and VI: group treated with all the minerals.

Values are expressed as Mean $\pm \mathrm{SD} ; n=5 .{ }^{\alpha} P<0.01$ when compared with group II, $\beta P<0.05$ when compared with group II, ${ }^{\rho} P<0.01$ when compared with group I, and $\gamma_{P}<0.05$ when compare with group I by Dunnette's multiple comparison test.

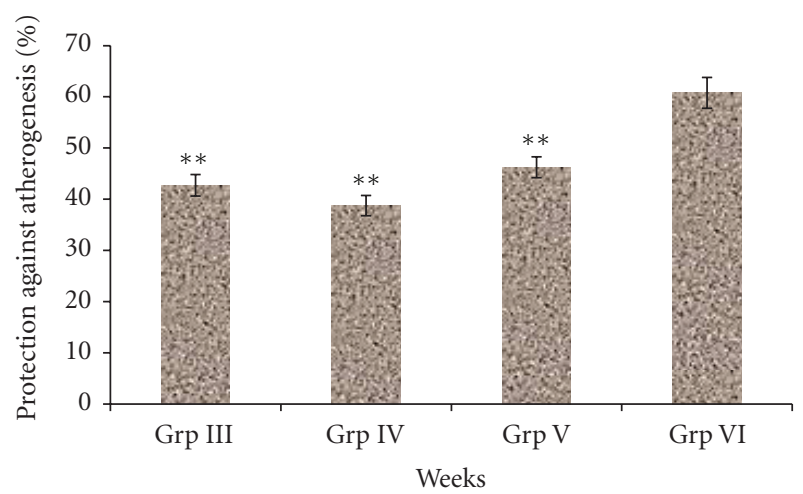

FIgURE 4: Percentage protection against atherogenesis of saltinduced hypertensive rats supplemented with minerals Grp I: normal untreated, Grp II: hypertensive untreated, Grp III: salt loaded treated with copper, Grp IV: salt loaded treated with manganese, Grp V: salt loaded treated with zinc, Grp VI: salt loaded treated with minerals combined ${ }^{* *} P<0.01$ when compared with grp VI.

Overproduction of superoxide anions and other free radicals due to activation of NADPH oxidase may overwhelm the antioxidant capability and cause imbalances between oxidant and antioxidant status which may result in oxidative stress. The result indicated that salt loading increased the arterial blood pressure of the rats and supplementation with antioxidant minerals prevents the elevation of blood pressure. The observation confirms the report that salt loading to various strains of rats such as Sprague-Dawley rats [20] and wistar rats [35] result in increased blood pressure.

The group supplemented with minerals combined nearly normalized the mean arterial blood pressure which indicates that combination therapy appears to be more effective in blood pressure reduction than single antioxidant mineral supplementation.

The blood pressure lowering effect of copper, manganese, and zinc in this model could be attributed to their free radical scavenging properties which decrease nitric oxide quenching 


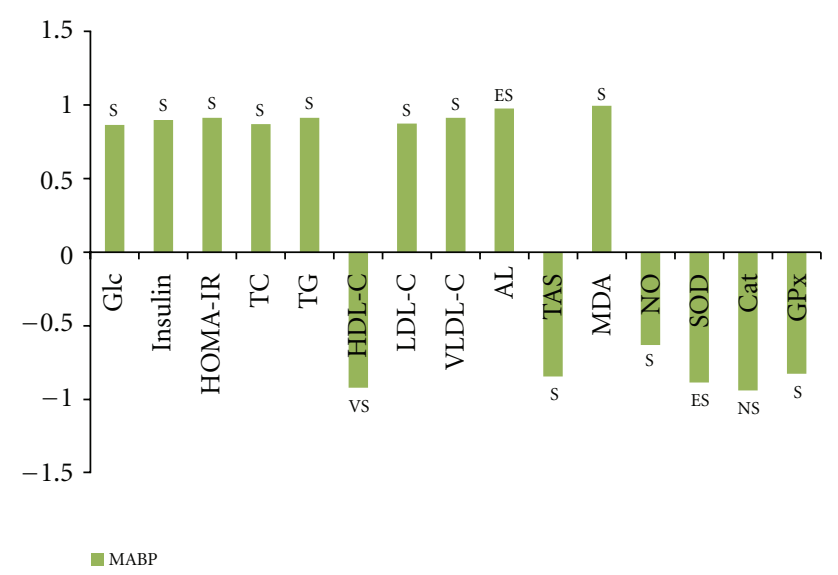

Figure 5: Correlation coefficient $(r)$ of MABP against glucose, insulin resistance, lipid profile, and oxidative stress markers. MABP: mean arterial blood pressure, Glc: glucose, HOMA-IR: Homeostasis Model Assessment-Insulin Resistance GPx: glutathione peroxidase, SOD: superoxide dismutase, TC: total cholesterol, TG: triglyceride, HDL-C: high-density lipoprotein cholesterol, LDLC: low density lipoprotein cholesterol, VLDL-C: very low-density lipoprotein cholesterol, AI: atherogenic index, vit: vitamin, TAS: total antioxidant status, MDA: malondialdehyde, NO: nitric oxide ES: extremely significant, VS: very significant, S: significant, and NS: not significant.

by superoxide anion, decrease NADPH oxidase activity, or increase superoxide dismutase activity since these minerals form an essential and integral part of superoxide dismutase. The result of the current work confirms the report that zinc supplementation lowers the blood pressure of salt-loaded hypertensive rats [36].

The increased levels of triglyceride, total cholesterol, low-density lipoprotein cholesterol, and very low-density lipoprotein-cholesterol and decrease in the high-density lipoprotein-cholesterol observed in hypertensive rats compared to the supplemented group corroborated with several studies $[37,38]$. The possible mechanism underlying the relationship between increased levels of HDL-C and decreased LDL-C and cardiovascular outcome of hypertension following supplementation in this study could be due to increased synthesis or decreased degradation of HDL-C that may decrease the oxidized lipid species in LDL particles, thereby protecting them from atherogenesis. This might indeed reflect the starting point for the protection against atherosclerosis. The result also showed decreased in the levels of glucose, insulin, insulin resistance, malondialdehyde, and increased nitric oxide of the supplemented groups as compared with hypertensive control. The improved endothelial function and insulin sensitivity observed in the supplemented groups confirms the role of antioxidant copper, manganese and zinc in the management of hypertension. Thus, the exact molecular mechanisms underlying antioxidant effects of these minerals on insulin sensitivity and endothelial function were not fully determined in this model but could be mediated through oxidative stress suppression which resulted in improving antioxidant status and endothelial function as evidenced by the decreased level of lipid peroxidation index, malondialdehyde, and increased nitric oxide, a measured of endothelial function.

The results of the study suggest that salt loading induces hypertension via oxidative stress, since it elicits lipid peroxidation and influence, the activities of antioxidant enzymes in the rats. This is indicated by the increase in the total antioxidant status and activities of superoxide dismutase, catalase, and glutathione peroxidase following supplementation with antioxidant minerals which was overwhelmed due to oxidative stress induced by salt.

Attempt was also made to correlate the mean arterial blood pressure with all the biochemical parameters assessed in our model in order to determine the degree of association between these variables. The result indicated significant positive correlation between mean arterial blood pressure and glucose, total cholesterol, triglyceride, low density lipoprotein-cholesterol, very low-density lipoprotein cholesterol, atherogenic index, insulin resistance, and malondialdehyde while high-density lipoprotein cholesterol, total antioxidant status, catalase, superoxide dismutase, and glutathione peroxidase showed significant negative correlation.

The positive correlation between MABP and malondialdehyde suggest that increase in blood pressure resulted in more production of thiobarbituric acid reactive substances, and supplementation decreased both MABP and MDA. The negative correlation between MABP and total antioxidant status, catalase, SOD, and glutathione peroxidase is evidence that increase in the MABP downregulated the activities of these enzymes and decreased the TAS which could be attributed to the production of excess free radicals, but supplementation ameliorate, the effects.

These observations further confirmed the role of oxidative stress in hypertension, and supplementations with antioxidant minerals have the potential to prevent or delay the cardiovascular complications of hypertension since our results provides antioxidant protection. However, it is not clear whether increase in the amount of reactive oxygen species is a consequence of hypertension or otherwise.

\section{Conclusion}

The result confirmed the role of oxidative stress in hypertension and underscores the role of copper, manganese, and zinc in delaying and treatment of cardiovascular complications of hypertension.

\section{References}

[1] C. J. L. Murray and A. D. Lopez, "Mortality by cause for eight regions of the world: global burden of disease study," Lancet, vol. 349, no. 9061, pp. 1269-1276, 1997.

[2] R. Rodrigo, W. Passalacqua, J. Araya, M. Orellana, and G. Rivera, "Implications of oxidative stress and homocysteine in the pathophysiology of essential hypertension," Journal of Cardiovascular Pharmacology, vol. 42, no. 4, pp. 453-461, 2003.

[3] K. Miyajima, S. Minatoguchi, Y. Ito et al., "Reduction of QTc dispersion by the angiotensin II receptor blocker valsartan may be related to its anti-oxidative stress effect in patients with 
essential hypertension," Hypertension Research, vol. 30, no. 4, pp. 307-313, 2007.

[4] B. R. Winkelmann, J. Hager, W. E. Kraus et al., "Genetics of coronary heart disease: current knowledge and research principles," American Heart Journal, vol. 140, no. 4, pp. S11-S26, 2000.

[5] B. Rassler, "The renin-angiotensin system in the development of salt-sensitive hypertension in animal models and humans," Pharmaceuticals, vol. 3, no. 4, pp. 940-960, 2010.

[6] S. Oparil, M. A. Zaman, and D. A. Calhoun, "Pathogenesis of hypertension," Annals of Internal Medicine, vol. 139, no. 9, pp. 761-776, 2003.

[7] E. Grossman, "Does increased oxidative stress cause hypertension?" Diabetes care, vol. 31, pp. S185-S189, 2008.

[8] K. Yasunari, K. Maeda, M. Nakamura, and J. Yoshikawa, "Oxidative stress in leukocytes is a possible link between blood pressure, blood glucose, and C-reacting protein," Hypertension, vol. 39, no. 3, pp. 777-780, 2002.

[9] F. Lacy, D. T. O'Connor, and G. W. Schmid-Schönbein, "Plasma hydrogen peroxide production in hypertensives and normotensive subjects at genetic risk of hypertension," Journal of Hypertension, vol. 16, no. 3, pp. 291-303, 1998.

[10] C. Berry, C. A. Hamilton, M. J. Brosnan et al., "Investigation into the sources of superoxide in human blood vessels: angiotensin II increases superoxide production in human internal mammary arteries," Circulation, vol. 101, no. 18, pp. 2206-2212, 2000.

[11] R. M. Touyz and E. L. Schiffrin, "Increased generation of superoxide by angiotensin II in smooth muscle cells from resistance arteries of hypertensive patients: role of phospholipase D-dependent NAD $(\mathrm{P}) \mathrm{H}$ oxidase-sensitive pathways," Journal of Hypertension, vol. 19, no. 7, pp. 1245-1254, 2001.

[12] Y. Taniyama and K. K. Griendling, "Reactive oxygen species in the vasculature: molecular and cellular mechanisms," Hypertension, vol. 42, no. 6, pp. 1075-1081, 2003.

[13] D. Versari, E. Daghini, A. Virdis, L. Ghiadoni, and S. Taddei, "Endothelium-dependent contractions and endothelial dysfunction in human hypertension," British Journal of Pharmacology, vol. 157, no. 4, pp. 527-536, 2009.

[14] M. Félétou, R. Köhler, and P. M. Vanhoutte, "Endotheliumderived vasoactive factors and hypertension: possible roles in pathogenesis and as treatment targets," Current Hypertension Reports, vol. 12, no. 4, pp. 267-275, 2010.

[15] Y. Hiroyuki, S. Masamichi, and W. Osamu, "Zinc deficiency and hypertension," in Proceedings of the 18th Symposium on Trace Nutrients Research, pp. 67-71, 2001.

[16] J. Zicha, Z. Dobešová, and J. Kuneš, "Relative deficiency of nitric oxide-dependent vasodilation in salt-hypertensive Dahl rats: the possible role of superoxide anions," Journal of Hypertension, vol. 19, no. 2, pp. 247-254, 2001.

[17] X. Chen, R. M. Touyz, J. B. Park, and E. L. Schiffrin, "Antioxidant effects of vitamins $\mathrm{C}$ and $\mathrm{E}$ are associated with altered activation of vascular NADPH oxidase and superoxide dismutase in stroke-prone SHR," Hypertension, vol. 38, no. 3, pp. 606-611, 2001.

[18] S. J. Duffy, N. Gokce, M. Holbrook et al., "Effect of ascorbic acid treatment on conduit vessel endothelial dysfunction in patients with hypertension," American Journal of Physiology, vol. 280, no. 2, pp. H528-H534, 2001.

[19] M. Boshtam, M. Rafiei, K. Sadeghi, and N. Sarraf-Zadegan, "Vitamin E can reduce blood pressure in mild hypertensives," International Journal for Vitamin and Nutrition Research, vol. 72, no. 5, pp. 309-314, 2002.
[20] N. Tian, K. D. Thrasher, P. D. Gundy, M. D. Hughson, and R. D. Jr Manning, "Antioxidant treatment prevents renal damage and dysfunction and reduces arterial pressure in saltsensitivity hypertension," Hypertension, vol. 45, no. 5, pp. 934939, 2005.

[21] P. Trinder, "Determination of blood glucose in blood using glucose oxidase with an alternative oxygen acceptor," Annals of Clinical Biochemistry, vol. 6, pp. 24-25, 1969.

[22] C. C. Allain, L. S. Poon, and C. S. G. Chan, "Enzymatic determination of total serum cholesterol," Clinical Chemistry, vol. 20, no. 4, pp. 470-475, 1974.

[23] N. W. Tietz, "Serum triglyceride determination," in Clinical Guide to Laboratory Tests, pp. 554-556, W.B. Saunders, Philadelphia, Pa, USA, 2nd edition, 1990.

[24] M. Burstein, H. R. Scholnick, and R. Morfin, "Rapid method for the isolation of lipoproteins from human serum by precipitation with polyanions," Journal of Lipid Research, vol. 11, no. 6, pp. 583-595, 1970.

[25] W. T. Friedewald, R. I. Levy, and D. S. Fredrickson, "Estimation of the concentration of low-density lipoprotein cholesterol in plasma, without use of the preparative ultracentrifuge," Clinical Chemistry, vol. 18, no. 6, pp. 499-502, 1972.

[26] R. D. Abbott, P. W. F. Wilson, W. B. Kannel, and W. P. Castelli, "High density lipoprotein cholesterol, total cholesterol screening, and myocardial infarction. The Framingham Study," Arteriosclerosis, vol. 8, no. 3, pp. 207-211, 1988.

[27] D. Koracevic, G. Koracevic, V. Djordjevic, S. Andrejevic, and V. Cosic, "Method for the measurement of antioxidant activity in human fluids," Journal of Clinical Pathology, vol. 54, no. 5, pp. 356-361, 2001.

[28] W. G. Niehaus and B. Samuelsson, "Formation of malonaldehyde from phospholipid arachidonate during microsomal lipid peroxidation," European Journal of Biochemistry, vol. 6, no. 1, pp. 126-130, 1968.

[29] D. R. Matthews, J. P. Hosker, and A. S. Rudenski, "Homeostasis model assessment: insulin resistance and $\beta$-cell function from fasting plasma glucose and insulin concentrations in man," Diabetologia, vol. 28, no. 7, pp. 412-419, 1985.

[30] S. Kadiri, "Tackling cardiovascular disease in Africa," British Medical Journal, vol. 331, no. 7519, pp. 711-712, 2005.

[31] T. Ogihara, T. Asano, K. Ando et al., "High-salt diet enhances insulin signaling and induces insulin resistance in Dahl saltsensitive rats," Hypertension, vol. 40, no. 1, pp. 83-89, 2002.

[32] B. M. S. Matthew, "Phenotypic expression of hypertension in rodent models through dietary manipulation," Research diets, pp. 1-3, 2008.

[33] P. Meneton, X. Jeunemaitre, H. E. De Wardener, and G. A. Macgregor, "Links between dietary salt intake, renal salt handling, blood pressure, and cardiovascular diseases," Physiological Reviews, vol. 85, no. 2, pp. 679-715, 2005.

[34] H. Kobori, A. Nishiyama, Y. Abe, and L. G. Navar, "Enhancement of intrarenal angiotensinogen in Dahl salt-sensitive rats on high salt diet," Hypertension, vol. 41, no. 3 I, pp. 592-597, 2003.

[35] S. Kagota, A. Tamashiro, Y. Yamaguchi et al., "Downregulation of vascular soluble guanylate cyclase induced by high salt intake in spontaneously hypertensive rats," British Journal of Pharmacology, vol. 134, no. 4, pp. 737-744, 2001.

[36] O. S. Adeniyi and A. A. Fasanmade, "Effect of dietary zinc supplementation on salt induced hypertension in rats," International Journal of Pharmacology, vol. 2, no. 5, pp. 485-491, 2006. 
[37] N. K. Lakshmana, J. Deepthi, Y. N. Rao, and K. M. Deedi, "Study of lipid profile, serum magnesium and blood glucose in hypertension," Biology and Medicine, vol. 2, no. 1, pp. 6-16, 2010.

[38] U. K. Biswas and A. Kumar, "A study on lipid profile, oxidation stress and carbonic anhydrase activity in patients with essential hypertension," Journal of Clinical and Diagnostic Research, vol. 4, no. 6, pp. 3414-3420, 2010. 


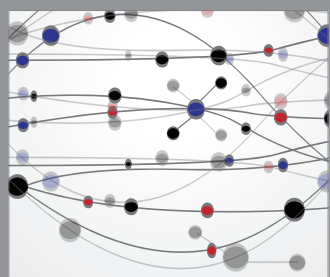

The Scientific World Journal
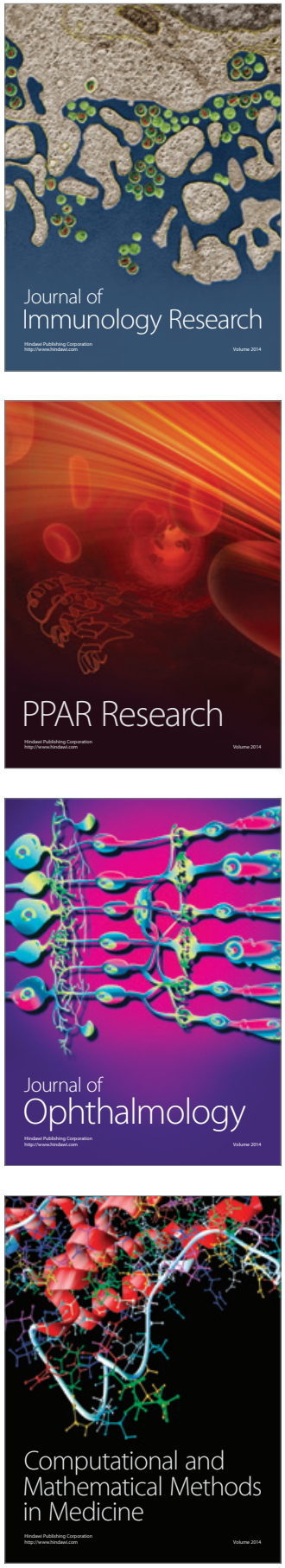

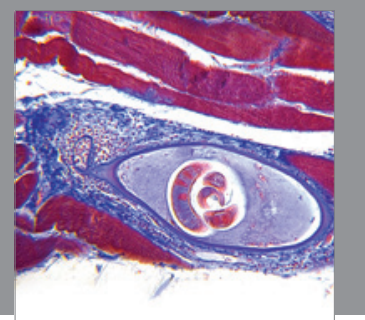

Gastroenterology

Research and Practice
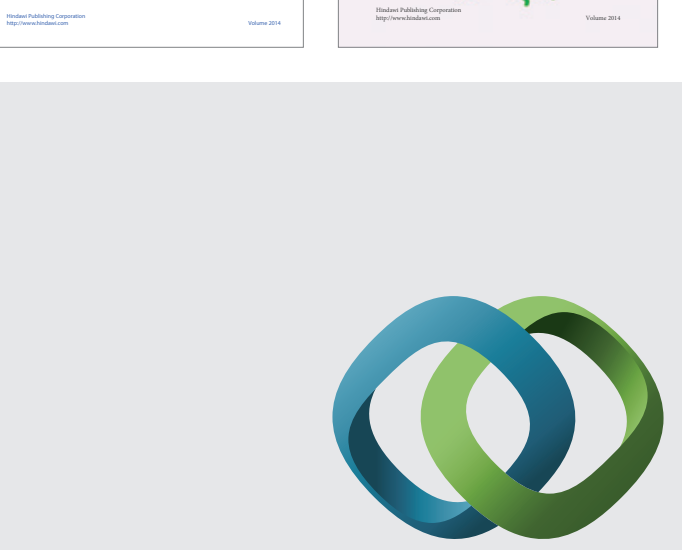

\section{Hindawi}

Submit your manuscripts at

http://www.hindawi.com
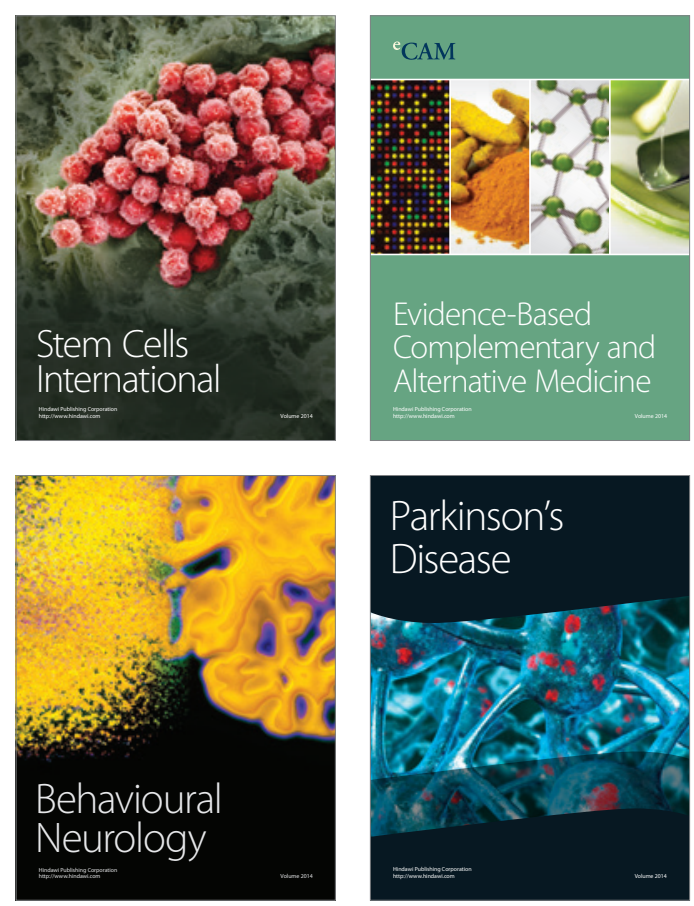

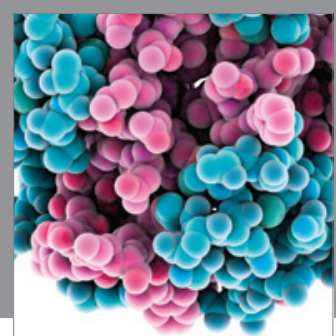

Journal of
Diabetes Research

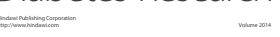

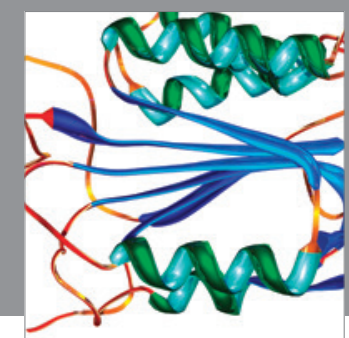

Disease Markers
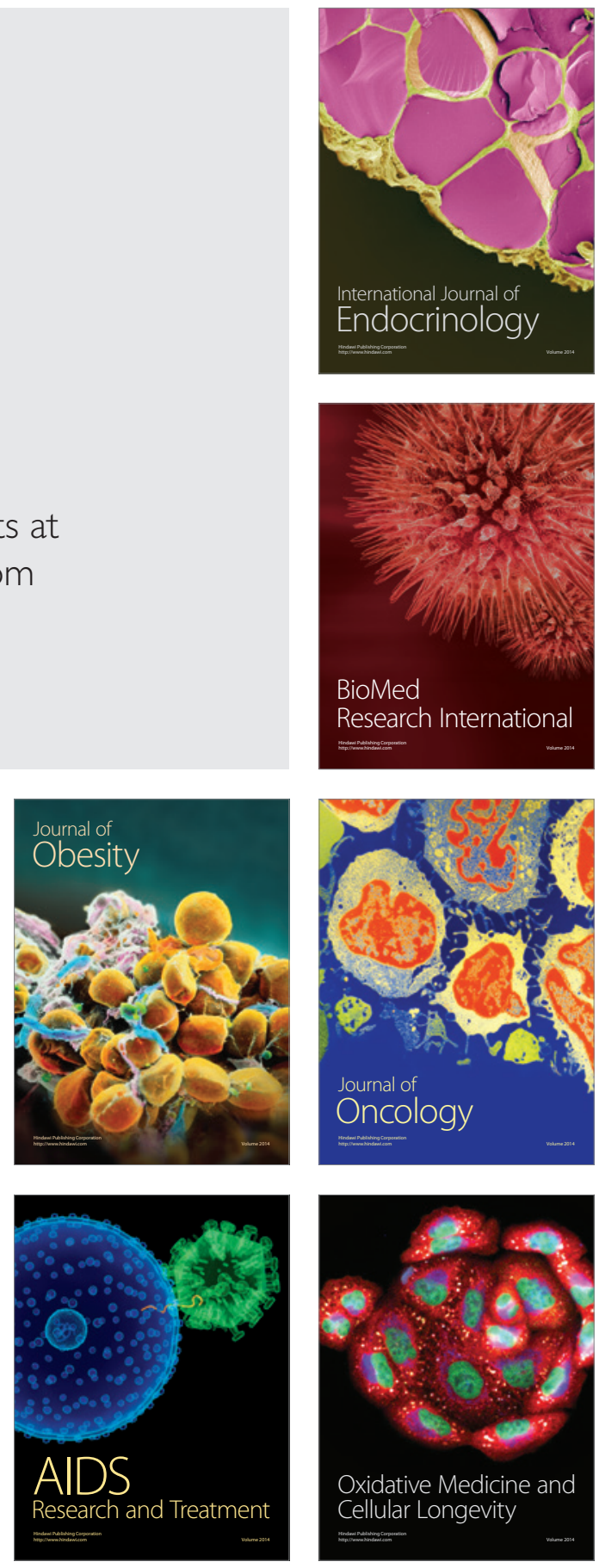